\title{
The plasticity of NBS resistance genes in sorghum is driven by multiple evolutionary processes
}

\author{
Emma Mace ${ }^{1 *}$, Shuaishuai Tai ${ }^{2}$, David Innes ${ }^{3}$, lan Godwin ${ }^{4}$, Wushu Huㄹ Bradley Campbell ${ }^{3}$, Edward Gilding $^{5}$, \\ Alan Cruickshank', Peter Prentis ${ }^{6}$, Jun Wang ${ }^{2,7,8}$ and David Jordan ${ }^{9}$
}

\begin{abstract}
Background: Increased disease resistance is a key target of cereal breeding programs, with disease outbreaks continuing to threaten global food production, particularly in Africa. Of the disease resistance gene families, the nucleotide-binding site plus leucine-rich repeat (NBS-LRR) family is the most prevalent and ancient and is also one of the largest gene families known in plants. The sequence diversity in NBS-encoding genes was explored in sorghum, a critical food staple in Africa, with comparisons to rice and maize and with comparisons to fungal pathogen resistance QTL.

Results: In sorghum, NBS-encoding genes had significantly higher diversity in comparison to non NBS-encoding genes and were significantly enriched in regions of the genome under purifying and balancing selection, both through domestication and improvement. Ancestral genes, pre-dating species divergence, were more abundant in regions with signatures of selection than in regions not under selection. Sorghum NBS-encoding genes were also significantly enriched in the regions of the genome containing fungal pathogen disease resistance QTL; with the diversity of the NBS-encoding genes influenced by the type of co-locating biotic stress resistance QTL.

Conclusions: NBS-encoding genes are under strong selection pressure in sorghum, through the contrasting evolutionary processes of purifying and balancing selection. Such contrasting evolutionary processes have impacted ancestral genes more than species-specific genes. Fungal disease resistance hot-spots in the genome, with resistance against multiple pathogens, provides further insight into the mechanisms that cereals use in the "arms race" with rapidly evolving pathogens in addition to providing plant breeders with selection targets for fast-tracking the development of high performing varieties with more durable pathogen resistance.
\end{abstract}

Keywords: Cereals, Disease resistance, Domestication, Maize, NBS-LRR genes, QTL, Rice, Selection, Sorghum

\section{Background}

The grasses, including the major cereals wheat, barley, maize, rice and sorghum, are the most agronomically and economically important species, collectively feeding over two thirds of the world population [1]. However the production of these crops is challenged by pathogens which pose a major threat to the global human food supply. At least $30 \%$ of global food production is lost to pathogens $[2,3]$ and the impact of disease outbreaks can be particularly acute in developing countries [4]. Amongst the cereals, sorghum which provides staple food for over 500 million people in the semi-arid tropics of Africa and Asia, in addition to being an important source of feed for

\footnotetext{
* Correspondence: emma.mace@daff.qld.gov.au

'Department of Agriculture, Fisheries \& Forestry (DAFF), Warwick, QLD, Australia

Full list of author information is available at the end of the article
}

livestock, is one of the best adapted to drought and high temperatures, and will play an increasingly important role in meeting the challenges of feeding the world's growing population. However, its productivity is often impacted by foliar fungal diseases. The most profitable and sustainable disease minimisation strategy is to grow genetically resistant varieties; consequently, selection for disease resistance is a critical component of nearly all plant breeding programs.

Among all disease resistance genes, the nucleotidebinding site plus leucine-rich repeat genes (NBS-LRR) are the most prevalent and ancient and are one of the largest gene families known in plants [5]. These genes are involved in the detection and response to diverse pathogens, including bacteria, viruses, fungi, nematodes, insects and oomycetes [5]. NBS-LRR genes encode an N-terminal variable domain, a central nucleotide-binding site (NBS) 
domain, and a C-terminal leucine-rich repeat (LRR) domain [6]. Further, classification based on the presence of an N-terminal Toll/interleukin-1 receptor (TIR) domain divides NBS-encoding genes into TIR and non-TIR subclass, though previous studies have shown that the TIR subclass is under-represented in the cereals, and in monocotyledonous plants in general $[7,8]$.

The most striking structural feature of the NBSencoding genes is the variable number of LRR domains, with some genes lacking LRR-coding domains completely [8]. These domains are highly variable regions thought to be responsible for recognising pathogen-encoded ligands [9]. In contrast, the NBS domain, involved in signalling, includes several highly conserved and strictly ordered motifs [10].

Previous studies have identified highly variable numbers of NBS-encoding genes across plant genomes, e.g. ranging from approximately 150 in arabidopsis [11] to almost 500 in rice [12], with sorghum reported as having between 211 [13] and 348 [14] NBS-LRR genes. It has been postulated that such rapid copy number evolution is driven by gene loss or expansion within a species through repeated cycles of duplication, divergence and eventual loss by pseudogene formation or deletion in response to diverse pathogens [15]. These genes are expected to be under continual selection pressure for alleles that allow the plant to defend against pathogen attack. Initial studies have shown that NBS-encoding genes are more often the target of selection than non-NBS-encoding genes [16], but these NBS-encoding genes show molecular evidence consistent with the action of different types of selection. Some evolve relatively slowly whereas others exhibit typical patterns associated with rapid evolution, including multiple and variable copy number, a high ratio of non-synonymous to synonymous substitutions and high levels of within species polymorphism [17].

The availability of the whole genome sequences of a number of cereal crops $[13,18,19]$ has given rise to a suite of new studies assessing genome-wide sequence polymorphism within species [20-22]. A recent resequencing study in sorghum [23] generated high-coverage $(>20 \times)$ data for a diverse group of 44 wild, weedy and cultivated genotypes, spanning the dimensions of geographic origin, crop management and subgroup/race. This study utilises this resource which provides new opportunities to explore the evolutionary plasticity and resulting variability in NBS-encoding genes in sorghum wild and weedy genotypes in contrast to cultivated genotypes with respect to 1 ) previously identified genomic regions under selection during domestication and improvement; 2) sorghum fungal pathogen disease resistance QTL and 3) ancestral gene families shared with maize and rice. Such insights will shed new light on how the NBS-encoding gene family became the foremost pathogen surveillance system in cereal genomes. It will additionally provide breeders with new knowledge and tools for estimating the richness of resistance germplasm and targeting specific genomic regions in order to utilise these resources more efficiently.

\section{Results}

Polymorphism patterns in NBS-encoding genes in sorghum A total of 346 NBS-encoding genes, with highly conserved NBS regions, were identified within the $\sim 700 \mathrm{Mb}$ sorghum genome, accounting for $\sim 1.2 \%$ of all predicted gene models in the sorghum reference genome [13] (Additional file 1: Table S1), comparable to the recent study in sorghum [14] which identified 348 NBS-encoding genes. Based on sequence similarity in the N-terminal and LRR domains, the 346 genes could be classified into 14 different NBS types (Table 1); of which 228 had LRR domains. The NBS-encoding genes were distributed unevenly across the genome (Figure 1), with over $60 \%$ located on 3 chromosomes (SBI-02, SBI-05 and SBI-08). Additionally, over two-thirds of the NBS-encoding genes (68.7\%) were located in clusters on the chromosomes (Additional file 2: Table S2). The NBS-encoding genes were significantly enriched in the regions of the genome containing fungal pathogen disease resistance QTL $\left(\mathrm{X}^{2} p\right.$-value 0.00272). Additionally, NBS-encoding genes were significantly enriched in regions of the genome identified as being

Table 1 The number of NBS-encoding genes per genome

\begin{tabular}{lllll}
\hline $\begin{array}{l}\text { Predicted } \\
\text { protein domains }\end{array}$ & Letter code & S. bicolor & Z. mays & O. sativa \\
\hline NBS-LRR type genes & & & & \\
NBS-LRR & NL & 133 & 26 & 113 \\
CC-NBS-LRR & CNL & 24 & 7 & 39 \\
NBS-LRR-CC & NLC & 1 & 1 & 2 \\
NBS-LRR-X & NLX & 12 & 1 & 2 \\
NBS-X-LRR & NXL & 43 & 0 & 3 \\
CC-NBS-LRR-X & CNLX & 3 & 2 & 1 \\
CC-NBS-X-LRR & CNXL & 12 & 0 & 0 \\
TIR-NBS & TN & 0 & 0 & 1 \\
Total & & 228 & 37 & 161 \\
NBS type genes & & & & \\
NBS & N & 64 & 69 & 252 \\
CC-NBS & CN & 11 & 21 & 72 \\
NBS-CC & NC & 1 & 0 & 7 \\
CC-NBS-X & CNX & 3 & 0 & 2 \\
NBS-CC-X & NCX & 4 & 0 & 0 \\
X-CC-NBS & XCN & 8 & 0 & 1 \\
X-NBS & XN & 27 & 10 & 8 \\
Total & & 118 & 100 & 342 \\
Grand total & & 346 & 137 & 503 \\
\hline & & & &
\end{tabular}




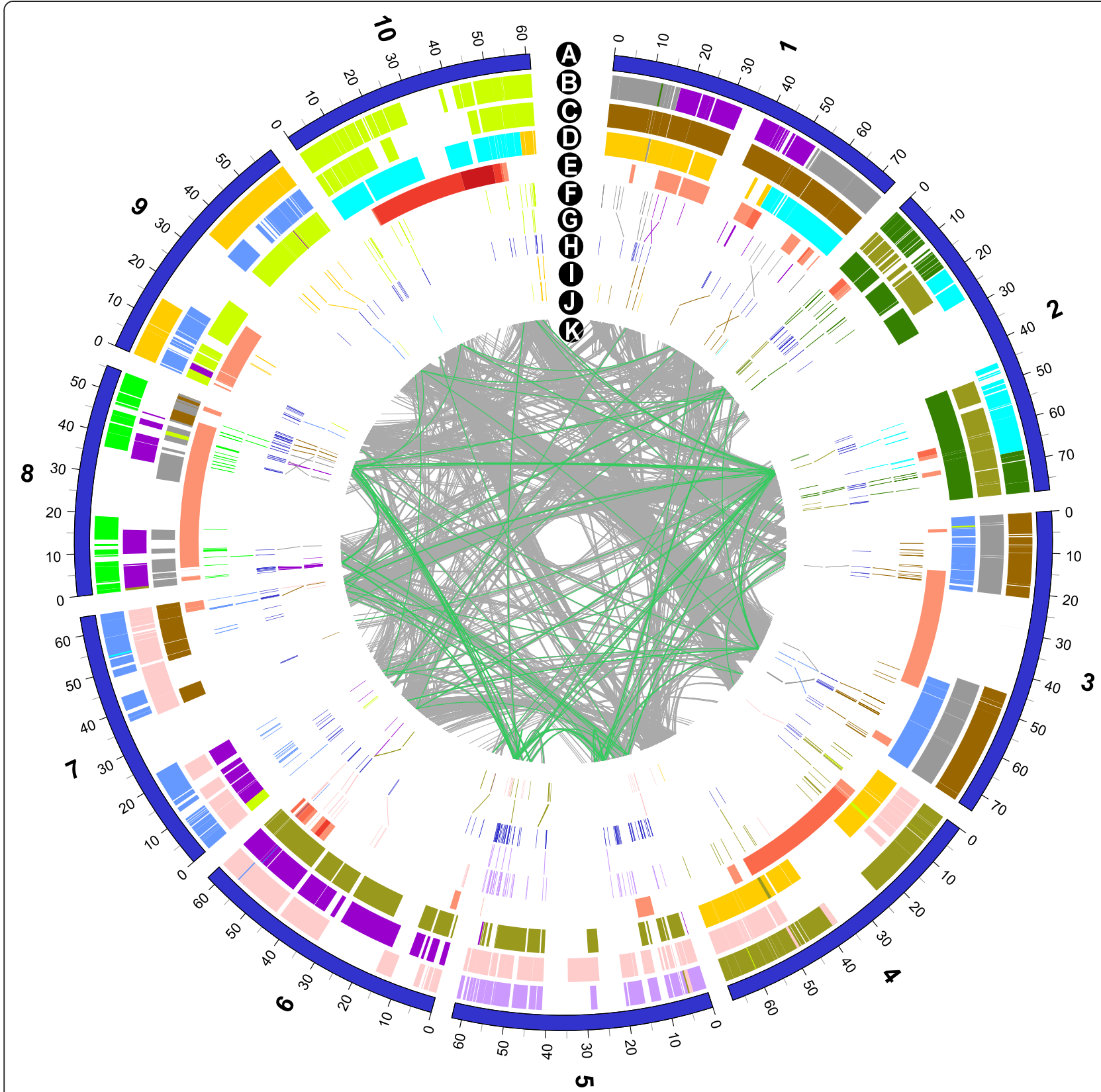

Maize Chromosomes C D ( )

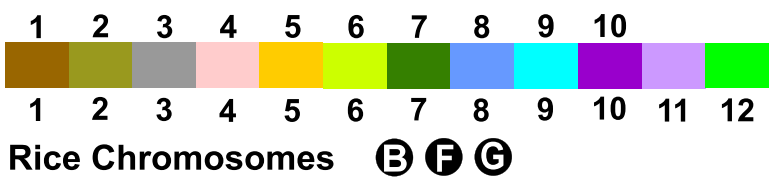

\section{QTL number $\mathbf{E}$}

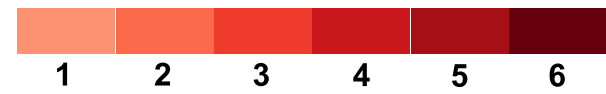

Figure 1 Comparison of genome-wide distribution of NBS-encoding genes in S. bicolor, Z. mays and O. sativa. Lanes detailed as follows; A: sorghum chromosomes, B: rice chromosomes, C, D: maize chromosomes, E: sorghum disease resistance QTL, F: rice NBS genes, G: homology between rice and sorghum NBS genes, H: sorghum NBS genes, I: homology between maize and sorghum NBS genes, J: maize NBS genes, $\mathrm{K}$ : duplicated genes in sorghum with NBS genes highlighted in green.

under purifying selection. This pattern was observed both through domestication $\left(\mathrm{X}^{2} p\right.$-value 0.000539$)$ and improvement $\left(\mathrm{X}^{2} p\right.$-value 0.0000046$)$, characterized by elevated differentiation between wild, landrace and improved groups and with low nucleotide diversity and negatively skewed allele frequency spectra [23]. 
NBS-encoding genes were also enriched in chromosome regions under balancing selection $\left(\mathrm{X}^{2} p\right.$-value $0.0323)$, with contrasting diversity and differentiation signatures to purifying selection. As a comparison, the distribution of the sorghum genes homologous to a set of 176 house-keeping genes identified in arabidopsis [24] were analysed and found not to be significantly enriched in the regions of the genome identified as being under purifying or balancing selection $\left(\mathrm{X}^{2} p\right.$-value 0.956 and 0.202 , respectively) or in the regions of the genome containing fungal pathogen disease resistance QTL ( ${ }^{2} p$-value 0.079).

Polymorphism patterns in the NBS-encoding genes were also distributed unevenly across the genome. Overall, NBS-encoding genes had significantly higher diversity $\left(P<3.18^{\mathrm{e}-9}\right.$ by paired t-test) in comparison to 346 randomly selected non NBS-encoding genes in the sorghum filtered gene set. NBS-encoding genes were significantly enriched in the upper $5 \%$ tail of the empirical distribution of the nucleotide diversity measure $(\theta \pi)$ for all genes in the sorghum filtered gene set $(n=29,346)$. This pattern was observed for all three groups $\left(\mathrm{X}^{2} p\right.$-value $\left.<0.0001\right)$ (Figure 2); wild and weedy, landraces and improved inbreds. Differences between genotype groups were also observed in the nucleotide diversity levels in the NBSencoding genes (Additional file 2: Figure S1) with diversity in the cultivated groups being consistently lower in comparison to the wild and weedy genotypes, an observation made previously genome-wide in both genic and non-genic regions [23]. A significant reduction, relative to all genotype groups, in NBS gene diversity, was only observed in the improved inbred group of sorghum genotypes (enrichment in the lower $5 \%$ tail of the empirical distribution of $\theta \pi ; \mathrm{X}^{2} p$-value 0.046) (Figure 2).

In total, just under $20 \%$ of all the NBS-encoding genes had patterns of molecular variation consistent with the action of selection, as measured by skewed diversity $(\theta \pi$ and $\theta \mathrm{w}$ ), allele frequency spectra (Tajima's D) and between group differentiation (FST) values. Just over half (38) of these NBS-encoding genes had signatures of purifying selection, with a drive towards beneficial allele fixation and selective removal of deleterious alleles through both natural and human-mediated selection. Eleven NBS-encoding genes were completely invariant in both the cultivated and wild groups, with a further 10 genes fixed only in the cultivated groups (Additional file 1: Table S1). The majority of these invariant genes $(86 \%)$ occurred in gene clusters. The mlHKA test was used to validate whether the NBS-encoding domestication and improvement candidates showed patterns of genetic variation consistent with positive selection [25]. A model of directional selection best explained the patterns of polymorphism to divergence of the 17 variant candidate genes for domestication and improvement relative to 38 neutral loci (mean log likelihood ratio test statistic $=372 ; P<0.0001$ for all comparisons; Additional file 2: Table S3). In contrast, 23 NBS-encoding genes had molecular signatures consistent with balancing selection, in which multiple alleles were maintained at intermediate frequencies in ancestral and descendant populations. The mlHKA test identified that a model of

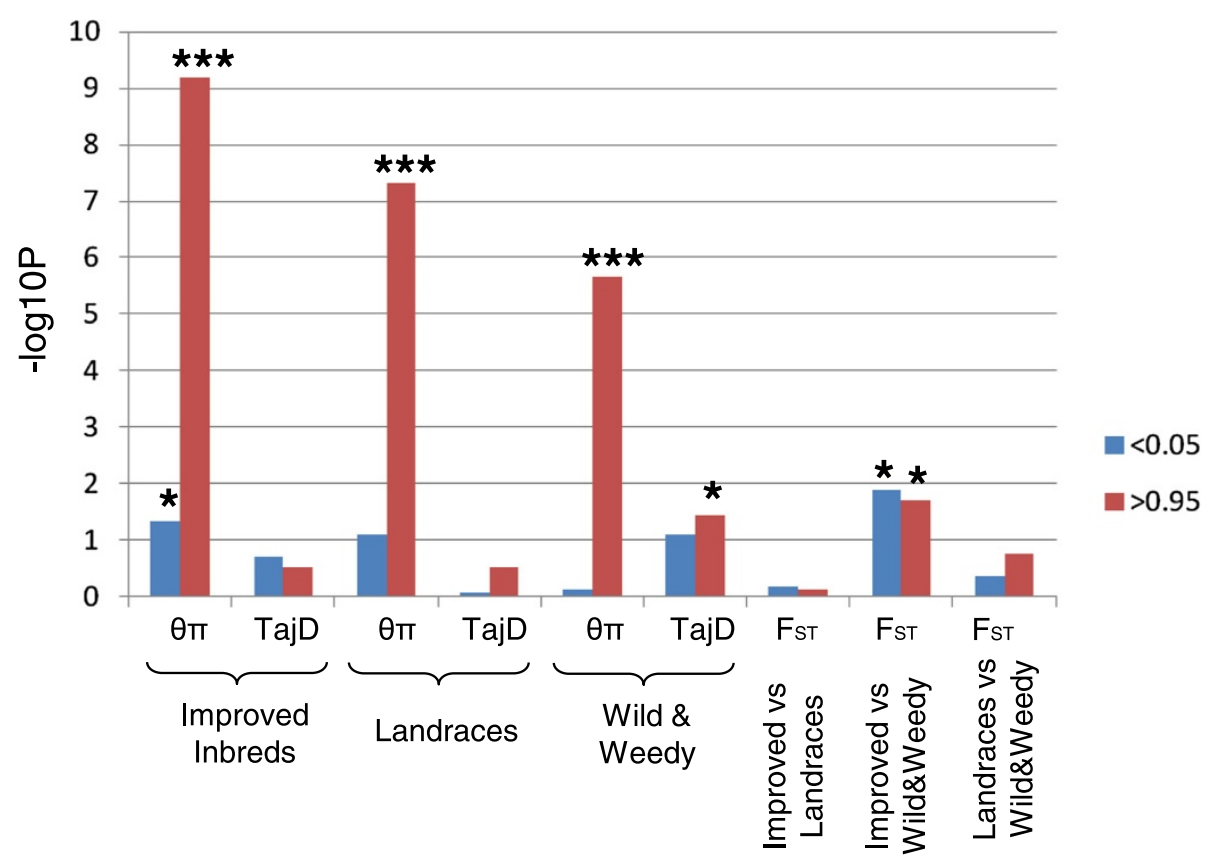

Figure 2 Enrichment of sorghum NBS genes in the upper and lower $5 \%$ tail of the empirical distribution of 3 different population statistics (Pi, TajD (Tajima's D). 
balancing selection best explained the patterns of genetic variation in these $23 \mathrm{NBS}$-encoding domestication and improvement candidates (mean log likelihood ratio test statistic $=493.2 ; P<0.0001$ for all comparisons; Additional file 2: Table S3). Over $90 \%$ of the 23 NBS-encoding genes with signatures of balancing selection had an LRR domain, in contrast to only $52 \%$ of the 38 NBS-encoding genes with signatures of purifying selection (Additional file 2: Figure S2). Overall, NBS genes with LRR domains were more diverse than NBS-encoding genes without LRR domains, both in cultivated $(\theta \pi=0.00278$ with LRR domains vs $\theta \pi=0.002203$ without LRR domains) and wild and weedy groups $(\theta \pi=0.00384$ with LRR domains vs $\theta \pi=0.00364$ without LRR domains). Diversity also increased with increasing numbers of LRR domains across the three groups (Additional file 2: Figure S3).

NBS-encoding genes were more diverse in both regions under purifying and balancing selection (Table 2), in contrast to non-NBS encoding genes with signatures of selection, providing evidence of diversity in the NBSencoding genes increasing subsequent to selection. The degree of diversity recovery subsequent to selection also varied according to the type of NBS-encoding gene, with the $\mathrm{N}$ class of NBS-encoding gene showing the largest amount of diversity recovery in the improved inbred group, the CNL class showing the largest amount of diversity recovery in the landrace group, and the $\mathrm{XN}$ class showing the largest amount of diversity recovery in the wild and weedy group (Additional file 2: Figure S4). Although in the minority, there were examples of NBSencoding genes having lower diversity than non-NBSencoding genes in regions under purifying selection. In all cases the NBS-encoding genes with lower diversity than non-NBS-encoding genes in regions under selection co-located with fungal pathogen biotic stress QTL, in particular anthracnose and rust resistance (Additional file 2: Figures S5-6).

The NBS-encoding genes identified with signatures of balancing selection had higher numbers of protein variants versus NBS-encoding genes under purifying selection ( 7.6 versus 4.3 , respectively), in line with expectations of maintenance of an excess of polymorphism under

Table 2 The nucleotide diversity $(\theta \pi)$ in NBS-encoding genes in comparison to non-NBS-encoding genes across cultivated and wild and weedy sorghum genotype groups in regions of the genome under balancing and purifying selection

\begin{tabular}{llll}
\hline Selection type & Gene type & Cultivated & $\begin{array}{l}\text { Wild and } \\
\text { weedy }\end{array}$ \\
\hline Balancing & NBS-encoding gene & 0.00675 & 0.00761 \\
Balancing & Non NBS-encoding gene & 0.00528 & 0.00499 \\
Purifying & NBS-encoding gene & 0.00023 & 0.00222 \\
Purifying & Non NBS-encoding gene & 0.00018 & 0.00169 \\
\hline
\end{tabular}

balancing selection. Additionally the Ka:Ks ratio test, which compares the number of non-synonymous substitutions (potentially adaptive amino acid replacement changes) with the number of synonymous substitutions (assumed to be evolving neutrally), provided further evidence of adaptive substitutions accumulating at higher frequencies in the NBS-encoding genes with signatures of balancing selection in contrast to NBS-encoding genes with signatures of purifying selection (Table 3), likely due to the more frequent occurrence of advantageous nonsynonymous mutations in comparison to neutral sites. In comparison, the Ka:Ks ratio values were consistently lower for nonNBS encoding genes throughout the genome, whether under selection or neutral.

Across all 346 NBS-encoding genes, the occurrence of non-functional alleles, either through frame-shifts or large effect SNPs (premature stop codons, start codon to nonstart codon, stop codon to non-stop, splice site), ranged from $2.17 \%$ to $86.95 \%$ across the sorghum genotypes, with the wild and weedy genotypes having higher frequencies in comparison to the cultivated groups (Additional file 2: Table S4).

The NBS-encoding genes identified with signatures of balancing selection also had higher overall proportions of non-functional alleles, in comparison to NBS-encoding genes evolving under purifying selection or neutral expectations (Additional file 2: Figures S7). In the majority of cases (19/23 genes under balancing selection), less than $50 \%$ of the genotypes were impacted by frame-shifts or large effect SNPs, with multiple functional protein variants still present. Non-functional alleles occurred very rarely in NBS-encoding genes under purifying selection. Four of the NBS-encoding genes in sorghum $(\sim 1.2 \%)$ appeared to be psuedogenes in the sense that all of the alleles were rendered non-functional by frame-shifts or large deletions ( $>50 \%$ of gene). One of these genes (Sb05g007560; NXL classification) also had a signature of balancing selection and was in the upper $1 \%$ tail of the empirical distribution of the nucleotide diversity measure $(\theta \pi)$ for all genes in the sorghum filtered gene set, across cultivated and wild sorghum genotypes. This gene did not co-locate with a previously identified fungal pathogen biotic stress resistance QTL; however, it was observed that a differentiation between types of fungal pathogen biotic stress resistance QTL co-locating with NBS-encoding genes under purifying or balancing selection occurred.

Table 3 The Ka:Ks ratio for NBS-encoding genes and non NBS-encoding genes in regions under selection versus regions not under selection

\begin{tabular}{llll}
\hline & $\begin{array}{l}\text { Under purifying } \\
\text { selection }\end{array}$ & $\begin{array}{l}\text { Under balancing } \\
\text { selection }\end{array}$ & $\begin{array}{l}\text { Not under } \\
\text { selection }\end{array}$ \\
\hline NBS-encoding gene & 0.459 & 0.705 & 0.653 \\
Non NBS-encoding & 0.122 & 0.318 & 0.347 \\
gene & & & \\
\hline
\end{tabular}


Almost $80 \%$ of QTL co-locating with NBS-encoding genes under purifying selection were associated with rust or anthracnose resistance. In contrast, over $80 \%$ of QTL co-locating with NBS-encoding genes with signatures of balancing selection were associated with ergot resistance, whereas only 1 QTL for rust and anthracnose did so.

To investigate signatures of selective sweeps, the genetic diversity of the genes in the $100 \mathrm{~kb}$ region flanking the NBS-encoding genes were analysed and found to be significantly reduced in comparison to genome-wide averages; with flanking genes enriched in the lower 5\% tail of nucleotide diversity for all three groups (Additional file 2: Figures S8). In total, sixty-nine NBS-encoding genes were located within $100 \mathrm{~kb}$ of previously identified candidate genes under selection in sorghum [23], including previously described domestication genes, e.g. LA1, involved in tiller angle [26] and $\mathrm{Rd}$, involved in pericarp colour [27].
Polymorphism patterns in orthologous, paralogous and novel NBS-encoding genes in sorghum

Phylogenetic trees, constructed for the NBS-encoding genes within and across sorghum, maize and rice species (Figure 3 and Additional file 2: Figures S9-11), allowed the identification of cross-species (orthologous) gene families. With a clade definition of nucleotide similarity $<70 \%$ between clades (the genes in a clade identified as a multigene family), 647 clades were identified; 137 being multigene family clades containing 404 NBS-encoding genes in total; 85 of which belonged to 20 ancestral gene families, pre-dating species divergence (Additional file 2: Figure S12). The highest proportion of ancestral genes was identified in maize $(21.1 \%)$, followed by sorghum (8.8\%) and rice (6.3\%). Ancestral gene families occurred predominately in gene clusters syntenic across species (67.1\%). Overall, NBS-encoding genes in sorghum with signatures of
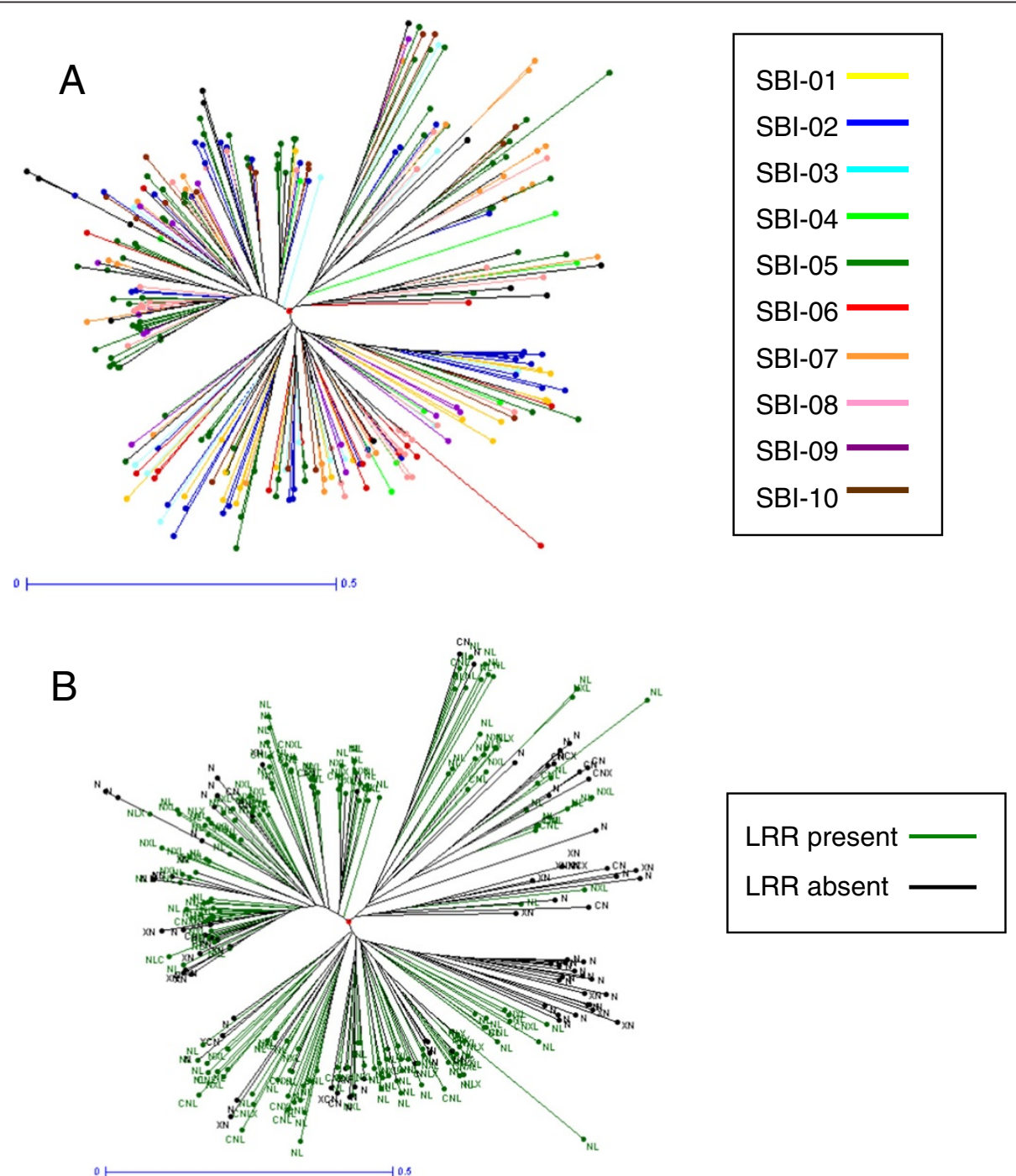

Figure 3 Phylogenetic trees of NBS-encoding genes from sorghum. A. Colour-coded by chromosome as indicated. B. Colour coded by presence or absence of LRR domain, as indicated, with gene letter codes as detailed in Table 1. 
selection (purifying or balancing) were more likely to be orthologous than neutral NBS-encoding genes, not under selection (Additional file 2: Figure S13). NBSencoding genes with signatures of balancing selection in sorghum had the highest proportion of ancestral gene family members in comparison to NBS genes that were neutral or under purifying selection.

A more detailed phylogenetic analysis of sorghum gene families, specifically, indicated that at 70\% nucleotide similarity and gene coverage, approximately one third of all NBS-encoding genes in sorghum belonged to paralogous, multi-gene families (Additional file 2: Figure S14). The majority $(76 \%)$ of the paralogous genes were located within the same gene clusters, although over $12 \%$ of the gene families had paralogues located across multiple chromosomes (Additional file 2: Figure S15). 55\% of paralogous NBS-encoding genes in sorghum also occurred within the recently duplicated super-gene regions of the genome, defined by 4DTv $<0.497$ [23]. Overall, higher diversity levels were observed in paralogous NBS-encoding genes $(\theta \pi=0.0039)$ in comparison to non-paralogous, singleton genes (0.0028; Additional file 2: Figure S16) and the proportion of paralogous NBS-encoding genes under selection was double that of singleton NBS-encoding genes in sorghum. Furthermore, the Ka:Ks ratio test identified a higher number of non-synonymous SNPs in paralogous NBS-encoding genes than in singleton genes (Additional file 2: Figure S17).

Syntenic selective sweeps across species were identified through comparisons of NBS-encoding genes under selection in rice [21] and maize [22] from previous studies. In total, from 1508 improvement candidate genes and 1764 domestication candidate genes identified from the analysis of resequencing data of 75 wild, landrace and improved maize lines [22], six maize NBS-encoding genes were identified as being under purifying selection (5 through improvement and 1 through domestication). These maize NBS-encoding genes under selection were orthologous with 5 sorghum NBS-encoding genes also identified as being under purifying selection, including the candidate gene for anthracnose resistance $S b C g 1$ on SBI-05. In a similar recent study in rice [21], which analysed resequencing data of 40 cultivated and 10 wild progenitors, 2506 candidate genes under artificial selection were identified, eight of which were NBSencoding genes. The rice NBS-encoding genes under selection were all located on chromosome 11 in 3 gene clusters, syntenous to three gene clusters on sorghum's SBI-05 including gene clusters containing $S b C g 1$ and candidate genes against Setosphaeria turcica, the causal agent of northern leaf blight disease in maize [28]. The sorghum gene pair St1A and St1B identified [28] belonged to one of the 20 ancestral gene families identified across sorghum, maize and rice, including the maize orthologue which was found to have upregulated transcripts after fungal challenge of Setosphaeria turcica. While the orthologous maize gene was not identified as being under selection [22], the rice and sorghum orthologues in this ancestral gene family have been previously identified as being under selection. In sorghum, two contrasting signatures of selection occur in the $S t 1 A$ and $S t 1 B$ gene pair, with St1A (Sb05g008280) having a signature of purifying selection and St1B (Sb05g008140) having a signature of balancing selection (Figure 4). A second ancestral gene family was also identified as being under selection across species, containing the maize Rp1-D gene (GRMZM5G879178), sorghum genes Rp1-dp3 (Sb03g036450) and rph1-3 (Sb08g002410) for rust resistance, and the rice Pi37 (Os01g57310) gene for resistance to rice blast disease. This was recently identified as a rapidly evolving gene family, termed "Rp1/Pi37" [17], which had an effector response that confers resistance to multiple pathogens across species.

The novel genes identified previously in sorghum [23] were enriched for NBS-encoding genes $\left(\mathrm{X}^{2} p\right.$-value 0.0051). In total, ten novel NBS-encoding genes were identified; all without an LRR domain (eight of which are classified as N, two as CN; Additional file 2: Table S5). Four novel genes were not present in any cultivated lines (Additional file 2: Figure S18); one was present only in S.propinquum, two only in the wild and weedy group and one in both $S$. propinquum and the wild and weedy group. Of the six novel genes that were present in at least one of the cultivated groups, one (novel_seq4_GLEAN_10000166) was present at high frequencies in all genotype groups except S. propinquum. All of the novel genes identified were in sorghum-specific gene families only.

\section{Discussion}

This study, focusing on the most prevalent and ancient of the disease resistance gene families, has presented new data demonstrating that NBS-encoding genes are under strong selection pressure in sorghum, through the contrasting evolutionary processes of purifying and balancing selection, and that they are enriched in the regions of the genome associated with fungal pathogen disease resistance. This study has also observed that NBS-encoding genes ancestral to cereals were less diverse than sorghum lineage-specific NBS-encoding genes and that the ancestral genes were more abundant in regions with signatures of selection (purifying or balancing) than in regions not under selection. This knowledge of the variation patterns of the different types of disease resistance genes indicates that NBS-encoding gene family members have real value for agriculture and can provide plant breeders with new tools to more effectively develop enhanced crop varieties with more durable resistance to plant fungal pathogens. 


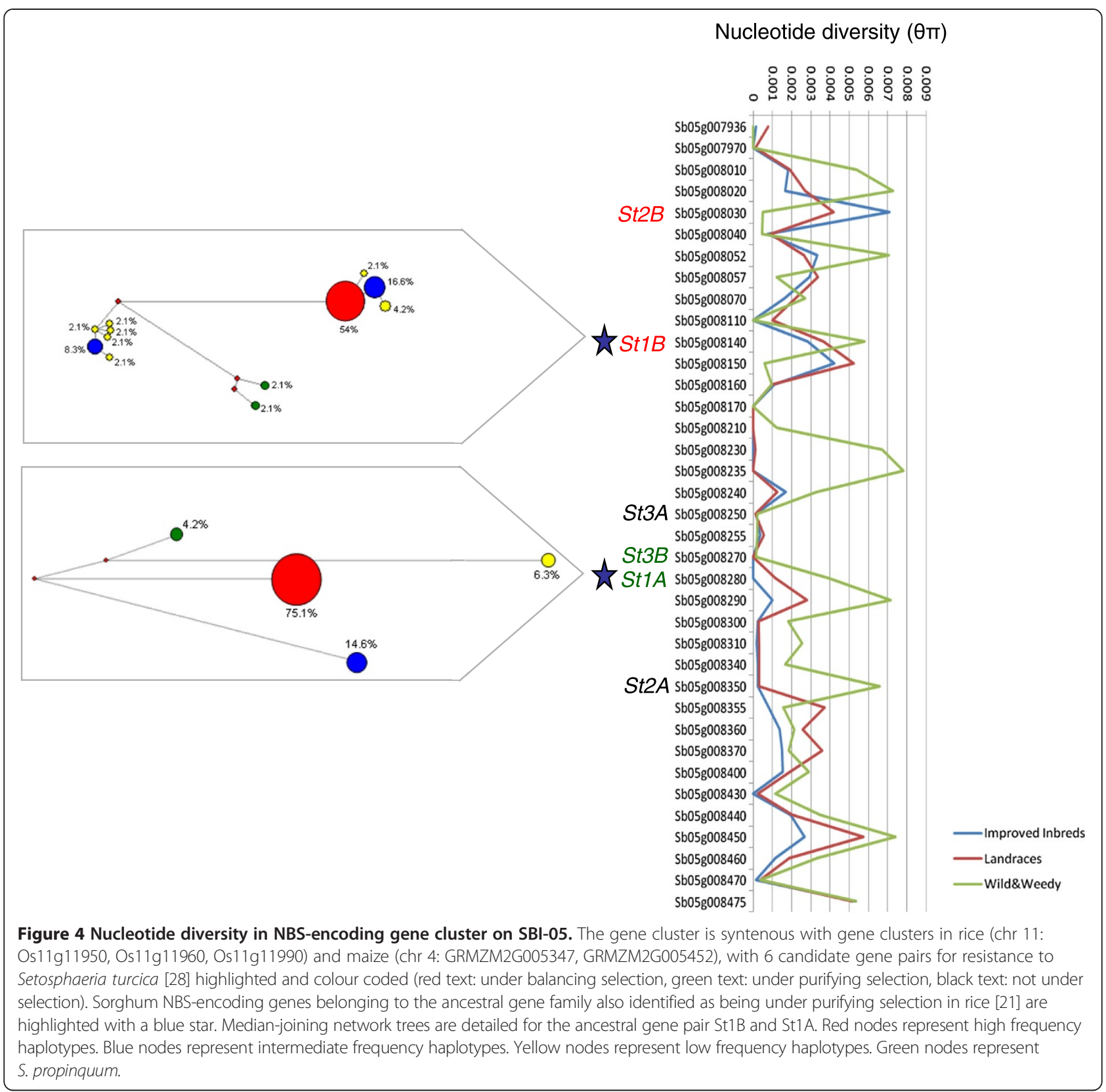

NBS-encoding genes have a role in the domestication of sorghum through contrasting mechanisms

The domestication syndrome is traditionally associated with traits such as tillering and seed shattering, with only limited studies to date supporting its association with disease resistance (e.g. [29]). The current study found that regions of the genome under purifying and balancing selection through both domestication and improvement were enriched for NBS-encoding genes. Two-thirds of the NBS-encoding genes under selection were associated with domestication rather than improvement which may have been influenced by the perennial nature of the wild relatives of sorghum, in contrast to the annual life-cycle of the majority of cultivated types, through increased plant longevity in the face of constant selection pressure for disease resistance. There was also evidence of NBS-encoding genes recovering and maintaining diversity more rapidly than non-NBS-genes in these regions. Only a few exceptions were observed, notably in NBS-encoding genes co-locating with QTL for resistance to anthracnose, which could reflect the variable selection pressures imposed by different pathogens. Additionally, an analysis of genomic regions under selection in a set of 539 advanced sorghum genotypes as part of the preliminary yield trials of the Australian sorghum pre-breeding program, based in Queensland, identified that the majority (58.3\%) of 
genomic regions under selection co-located with NBSencoding genes (data not shown).

Despite $\sim 12 \%$ of the NBS-encoding genes in sorghum having signatures of purifying selection, with concordant reduction in diversity, overall NBS-encoding genes had three times more nucleotide diversity than nonNBS-encoding genes $(\theta \pi=0.0031$ versus $\theta \pi=0.0018$, respectively) with an additional $\sim 8 \%$ showing specific signatures of balancing selection. Such heterogeneity of the impact of selection on NBS-encoding genes has been previously reported [5] with the NBS domain being more commonly subject to purifying selection, in contrast to the more variable LRR domain. This likely reflects the role of the LRR domain in recognition of constantly evolving pathogen ligands and a role for the NBS domain in recognition signalling [30]. The results of the current study are in-line with this finding and have demonstrated that while over $90 \%$ of the NBS-encoding genes with a signature of balancing selection had an LRR domain, this was reduced to only $50 \%$ in the NBS-encoding genes under purifying selection. The observed increase in nucleotide diversity with a concomitant increase in the number of LRR domains per gene emphasizes the highly variable nature of the LRR proteins.

\section{An integrated fungal pathogen disease-QTL map and NBS-encoding genes reveals regions of the sorghum genome associated with multiple quantitative disease resistance traits}

Regions of the genome containing fungal pathogen related biotic stress resistance QTL were found to be significantly enriched for NBS-encoding genes. We also found that the diversity of the NBS-encoding genes can vary according to the particular type of co-locating biotic stress resistance QTL, e.g. NBS-encoding genes underlying anthracnose QTL were significantly less diverse than the mean diversity of NBS-encoding genes in cultivated sorghum $(\theta \pi=0.0007$ versus $\theta \pi=0.0026$, respectively).

Previous studies investigating the coincidence of NBSencoding genes and disease resistance QTL in other species including rice [31] and soybean [32] also found a significant enrichment of the NBS-encoding genes in the QTL fraction of the genome. Approximately $36 \%$ of the sorghum genome was implicated in quantitative disease response (QDR) to fungal pathogens, less than reported recently for rice, 54\% [31]. Furthermore, nearly half of the total QDR genomic space consisted of colocalising QTL for the same trait, indicating the robustness of the disease resistance QTL identified. The majority $(>90 \%)$ of co-localising QTL conditioned resistance to multiple diseases, with a stand-out hotspot region on the long arm of SBI-06, containing 15 QTL for 6 traits from 4 studies [33-36]. Such hotspots for multiple disease resistances have been noted in other species, including maize [37], rice [31], and potato [38]. It is thought this could be due to single gene effects, whereby the resistance gene and QTL are allelic, or by the effects of clusters of genes. There were 7 NBS-encoding genes in the disease QTL hot-spot region on SBI-06; 2 singletons and 1 cluster of 5 genes, indicating that either individual genes can provide resistance against multiple pathogens or alternative resistance mechanisms are involved. Members of the defense-associated transcriptor family WRKY $(n=69)$ and MYB genes $(n=110)$ were investigated for the correspondence with fungal pathogen related biotic stress QTL in sorghum, however these genes were not found to be significantly enriched $\left(\mathrm{X}^{2} p\right.$-value 0.21 and 0.052 , respectively) in the QDR genomic space. Moreover the distribution of the sorghum genes homologous to a set of 176 house-keeping genes identified in arabidopsis [24] was compared to the QDR genomic space and also found not to be significantly associated.

\section{Deleterious mutations and presence/absence variations contribute to the rapid variation in NBS-encoding genes in the grasses}

Similar to previous findings $[13,39,40]$, the number of NBS-encoding genes in rice (503) was almost 4 times higher than in maize (137) and one and a half times higher than sorghum (346; Table 1), with no TIR-encoding NBS genes identified in sorghum, rice or maize [39,41]. Temporal difference in NBS-encoding gene expansion among species was estimated by examining the proportion of multi-gene families across similarity/coverage thresholds (Additional file 2: Figure S19). In line with recent findings [15], considerably smaller proportions of NBS-encoding genes were found between $60 \%$ and $80 \%$ similarity in maize, in comparison to sorghum and rice, indicating that recent duplications were likely to have dominated the NBS-encoding genes in the maize genome with more ancient duplications observed in sorghum and rice. A high degree of clustering was also a significant feature of the NBS-encoding genes across the three genomes, with rice having proportionally more NBS-encoding genes in clusters $(72.9 \%)$, potentially due to a higher number of localised tandem repeats, in comparison to sorghum $(68.7 \%)$ and maize (51.4\%). Of the defined gene clusters across genomes, maize was $100 \%$ syntenic across species, in contrast to sorghum (75.6\%) and rice, which had the lowest proportion of syntenic gene clusters (58\%) (Additional file 2: Figure S20). Rice had almost twice as many species-specific genes in clusters (72.4\%) in comparison to sorghum (42.8\%) and maize (43.4\%) (Additional file 2: Figure S21). Interestingly, the NBSencoding genes with signatures of balancing selection in sorghum were less likely to be located in clusters than NBS-encoding genes with signatures of purifying selection or those evolving neutrally (61\%, versus $92 \%$ 
versus $70 \%$, respectively), potentially indicating a fitness cost of NBS-encoding genes. The high proportion of NBS-encoding genes with signatures of purifying selection that were located in clusters provides further support for lineage specific rearrangements driving rapid evolution and the fixation of beneficial alleles. Such lineage-specific tandem duplications have led to higher numbers of gene copy variants in rice, in comparison to sorghum and maize, in line with the recent findings [40]. This highly dynamic clustering, through lineage specific rearrangements, is potentially a key mechanism driving the plasticity of NBS-encoding genes in the grasses via presence/absence variation (e.g. gene loss events, psuedogenization and novel genes) and copy number variation.

It has also been previously noted that the plasticity of disease resistance genes in plants can be mediated by gene regulators, including microRNAs (miRNA) [40]. miRNAs have been shown to play an important regulatory role in the growth and development of eukaryotes [42]. Specifically, they have been shown to regulate the expression of a number of key stress-related genes in plants (e.g. [43]). Amongst the cereals, sorghum and rice have higher percentages of NBS-encoding genes targeted by miRNA (37.5 and $36.4 \%$ respectively) in comparison to maize (28.94\%) [40]. It is possible that such high proportions of miRNA targeting disease resistance genes may be associated with increased functional redundancy in rice and sorghum in contrast to maize, through higher proportions of species-specific NBS-encoding gene copies; $84.6 \%$ in rice, $71.3 \%$ in sorghum and $52.9 \%$ in maize. It has been speculated that miRNAs have a role as a dosage regulator, through expression-level repression following large or local genome duplication events [40].

Previous studies looking at the numbers of NBSencoding genes across grass species have noted the rapid nucleotide evolution at the NBS-loci and the greater tendency for gene loss or gene number variation in contrast to house-keeping genes [15,39]. It has been postulated [15] that natural selection could be responsible for the drastic variation in numbers of NBS-encoding genes across rice, maize, sorghum and brachypodium, where the rapid expansion and/or contraction is a fundamentally important strategy for a species to adapt to a quickly changing spectrum of pathogens. The high frequency of NBS-encoding genes with non-functional alleles could indicate that there is a fitness cost associated with the disease resistance genes. For example, NBS-encoding genes that do not have useful functions in an environment lacking specific pathogens would more likely be lost or become pseudogenes through loss of function mutations to avoid a fitness cost. In the majority of cases the fixation of the null alleles was not observed, however in one case a null allele, caused by a premature stop SNP, in Sb08g005620 was found to be fixed across the cultivated genotypes (Additional file 2: Figure S22). This gene also had a signature of balancing selection however the fixation of the null allele in the cultivated lines could indicate the dual roles of both purifying selection to drive an increase in the frequency of new favourable mutations and balancing selection to maintain the different alleles.

It has been speculated previously [15] that multi-gene families facilitate the rapid evolution of NBS-encoding genes via frequent sequence exchange to generate novel gene sequences that may encode altered specificities. Our findings of increased polymorphism, and in particular increased non-synonymous polymorphisms (Ka:Ks ratio in cultivated sorghum of multi-gene families of 0.74 versus only 0.57 in the singleton genes in multi-gene families) support this hypothesis. The finding that the NBS-encoding genes from cross-species families were more abundant in regions under selection in sorghum supports previous results (e.g. [17]) that a diverse repertoire of NBS-encoding genes that provide resistance against rapidly evolving pathogens occurs not only within species but also across species.

\section{Complex evolutionary dynamics in the evolution of NBS-encoding genes identified across species}

Although there have been numerous studies characterising NBS-encoding genes across species, to date there have been limited comparable studies characterising NBS-encoding gene polymorphisms within species. Existing studies also report higher nucleotide diversity in NBS-encoding genes in arabidopsis [16] and rice (e.g. [12,21,44]) in comparison to genome-wide values. A study in arabidopsis [16] focused specifically on the evolutionary dynamics of a subset of 27 NBS-encoding genes by sequencing the LRR domain in 96 A. thaliana accessions, and observed a continuum of possible states in the evolutionary processes, including selective sweeps and balancing selection with many stages in between. Although several loci could be identified as candidates for recent selective sweeps, they found that this scenario was not common and overall found limited evidence for selective sweeps and hence, did not support the co-evolutionary arms-race hypothesis as a general evolutionary model for this group of genes. Additionally, only weak evidence was found for signatures of balancing selection acting to maintain multiple alleles at intermediate frequencies over prolonged periods of time. However, a recent genome-wide resequencing study in rice [21], looking at a subset of 102 NBS-encoding genes, found some evidence to support selective sweeps in cultivated rice, with 23 NBS-encoding genes showing significantly lower diversity than the genome average. The dynamic nature of a larger set of 725 NBS-encoding genes in rice was also demonstrated in a recent study [44], which showed that presence/absence polymorphism, caused by 
frequent deletions and translocations, were prevalent between different accessions of rice, in addition to across grass species. Another study in rice [12] also identified a high frequency of presence/absence variation in a subset of NBS-encoding genes between 21 cultivated and 14 wild rice populations, and postulated that such variation could potentially result from geographic differentiation. Pathogen prevalence and virulence is undoubtedly impacted by geographic differentiation and hence such expansion and/or contraction in the number of NBSencoding genes appears to be an important strategy for many cereal species to adapt to the quickly changing spectrum of pathogens. Segmental and tandem duplications and gene conversion are likely to have contributed to the high degree of clustering of NBS-encoding genes across species (e.g. [40]), further leading to synteny erosion and gene loss. The current study also identified presence/ absence variation in $~ 5 \%$ of all the NBS-encoding genes in sorghum, with 10 ten novel NBS-encoding genes identified, in addition to 8 genes with gene-loss events observed. This demonstrates the on-going dynamic and highly plastic nature of the largest of the disease resistance gene families.

\section{Conclusions}

A key mechanism driving the rapid variation in NBSencoding genes in the cereals is the highly dynamic clustering, through lineage specific rearrangements via presence/ absence variation (e.g. gene loss events, psuedogenization and novel genes) and copy number variation. Multiple evolutionary processes drive the plasticity of the NBS-encoding genes in sorghum; with the nucleotide sequence summary statistics depicting a continuum of possible states in the evolutionary process including both purifying and balancing selection. Such contrasting evolutionary processes have impacted ancestral genes, across the cereals, more than species-specific genes in sorghum.

As plant breeders seek to identify and deploy robust disease resistances, this study provides them with a clear understanding of the origins and allelic diversity of this rich gene family so important to the past, present and future of sorghum, a staple food crop for half a billion people. More broadly, by understanding the mechanisms that cereals use in the "arms race" with rapidly evolving fungal pathogens through NBS-encoding gene family expansion via duplication and rearrangements, researchers and breeders are better equipped to effectively manipulate the plants defenses in this continuing battle.

\section{Methods}

\section{Identification of NBS-encoding genes}

Genome assemblies and predicted gene models for sorghum (v1.4) [13], maize (v1.0) [19] and rice (O. sativa subsp. japonica; Release 7) $[18,45]$ were obtained from JGI (http://www.phyotozome.net/x), Maizesequence. org (http://ftp.maizesequence.org/current/filtered-set/) and MSU (ftp://ftp.plantbiology.msu.edu/pub/data/Eukaryotic_ Projects/o_sativa/annotation_dbs/pseudomolecules/version 7.0/all.dir/) respectively. To identify NBS-encoding genes in the three grass species, BLASTp with the amino acid sequence of the NB-ARC domain (Pfam: PF00931) was used; the threshold expectation value was filtered on 104. Subsequently the Pfam (Protein family) database was used to determine whether the corresponding candidate NBS protein encoded TIR, NBS or LRR motifs. Each of the candidate genes was subsequently checked manually by using existing annotations in GenBank to confirm that they encoded the expected NBS proteins. COILS under a threshold of 0.9 was then employed to specifically detect $\mathrm{CC}$ (coiled coils) domains. In each species genome, a gene cluster was defined if two or more NBS-encoding genes were located within $200 \mathrm{~kb}$ [46].

\section{Sequence alignment and phylogenetic analysis}

Multiple alignments of the predicted amino acid sequences of the conserved NBS domain (PFAM00931) were performed by ClustalW [47] and Mega5.0 [48]. The extent of nucleotide divergence and gene coverage were calculated to identify gene families between all identified NBS-encoding genes both within each species and across all three species using a previously described perl script [4]. Phylogenetic trees were constructed using the aligned nucleotide sequences of the NBS protein domain (PFAM00931) in TreeBest based on the neighbour-joining method. The tree was displayed using DARwin5.0 software [49]. Syntenic regions of the whole genomes of sorghum, rice and maize [50], with the locations of the identified NBS-encoding genes highlighted, was displayed using the software Circos [51].

\section{Data Analysis}

The sequence data of all of the identified NBS-encoding genes in sorghum were extracted from the whole genome resequencing data generated across 44 sorghum genotypes [23], representing three groups (wild and weedy group, landrace group and improved inbred group) (Additional file 3: Table S7). The following summary statistics were calculated as previously described: the average pairwise divergence within a group $(\theta \pi)$, the Watterson's estimator $(\theta \mathrm{w})$ and Tajima's $\mathrm{D}$ were estimated for the identified NBS-encoding genes and the surrounding $10 \mathrm{~kb}$ genomic interval of the three groups were calculated using a BioPerl module and an in-house perl script. $\mathrm{F}_{\mathrm{ST}}$ was calculated, based on the same windows, to measure population differentiation using another BioPerl module. Summary statistics involving coding regions included numbers of synonymous (Ks) and nonsynonymous (Ka) substitutions, were calculated utilising the KaKs_Calculator1.2 
software (MYN method) [52], and the number of protein variants based on nonsynonymous substitutions.

Regions of the genome under purifying selection were previously identified using the population genetics summary statistics $\left(\theta \pi, \theta \mathrm{w}\right.$, Tajima's $\mathrm{D}$ and $\left.\mathrm{F}_{\mathrm{ST}}\right)$ in the following three population pairwise comparisons: (i) wild and weedy versus landraces to identify domestication events, (ii) landraces versus improved inbreds to identify improvement events, (iii) wild and weedy versus improved inbreds to identify both domestication and improvement events. NBS-encoding genes with signatures of purifying selection were identified from the candidate genes previously described [23] in addition to the identification of NBSencoding genes in the top and bottom $5 \%$ tails of the empirical distribution of the population summary statistics. The population genetics summary statistics were also used to identify signatures of balancing selection with the following criteria; $\theta \pi$ and $\theta \mathrm{w}$ in the upper $25 \%$ of the empirical distribution; Tajima's D in the upper $5 \%$ of the empirical distribution and $\mathrm{F}_{\mathrm{ST}}$ values $<90 \%$ of the population pairwise distribution. Median-joining networks were constructed for selected NBS-encoding genes separately in Network [53]. A set of 17 of the non-invariant candidate genes under purifying selection were used as input, together with 38 neutral genes, for the mlHKA test for validation purposes [25]; in addition to a set of 23 candidate genes under balancing selection, together with the same set of 38 neutral genes. The mlHKA program was run under a neutral model, where numselectedloci $=0$, and then under a selection model, where numselectedloci $>0$. Significance was assessed by the mean log likelihood ratio test statistic, where twice the difference in log likelihood between the models is approximately chi-squared distributed with $\mathrm{df}$ equal to the difference in the number of parameters. Duplicated gene pairs were identified using the four-fold degenerate transversion (4DTv) ratio calculated previously [23].

Presence/absence patterns were identified across the 44 sorghum genotypes resequenced. BLASTp analysis with the amino acid sequence of the NB-ARC domain (Pfam: PF00931) was used for all 101 novel genes identified previously [23]; as previously the threshold expectation value was filtered on 10-4. Additionally the gene loss events were identified using read depth at $100 \mathrm{bp}$ resolution from all identified NBS-encoding genes in across all genotypes, as described previously [23].

\section{An integrated disease-QTL map}

26 sorghum fungal pathogen resistance QTL were retrieved from the set of 771 QTL projected onto the sorghum consensus map [54]. An additional 40 fungal pathogen disease resistance QTL and/or major effect genes from 4 additional studies $[35,36,55,56]$ were also projected onto the sorghum consensus map following the same strategy. The physical locations of a total of 66 fungal pathogen disease resistance QTL representing 9 traits from 11 studies (Additional file 2: Table S6) were predicted using the framework map of 504 sequenced markers with known genetic linkage distances, as detailed previously [54].

\section{Availability of supporting data}

The data sets supporting the results in this article are available from Dryad: doi:10.5061/dryad.d334b.

\section{Additional files}

Additional file 1: Table S1. Detailing the location, type and selection status of 346 NBS-encoding genes identified in sorghum.

Additional file 2: Contains 22 supplementary figures and 5 supplementary tables, all referenced in the main text.

Additional file 3: Table S7. Detailing the sorghum genotypes included in the current study.

\section{Abbreviations}

NBS-LRR: Nucleotide-binding site plus leucine-rich repeat; QTL: Quantitative trait loci; TIR: Toll/interleukin-1 receptor; QDR: Quantitative disease response; miRNA: microRNAs.

\section{Competing interests}

The authors declare that they have no competing interests.

\section{Authors' contributions}

ESM, SST, DJI and WSH undertook the data analysis; ESM, IDG, BCC, EKG, PJP, $A C$ and DRJ wrote the manuscript; ESM and DRJ conceived and designed the project; ESM, DRJ, AC and JW managed the project. All authors read and approved the final manuscript.

\section{Acknowledgements}

We would like to acknowledge statistical support provided by Colleen Hunt and preliminary sequence analysis provided by Sylvia Malory. We acknowledge funding support from the University of Queensland, the Department of Agriculture, Fisheries and Forestry, the Grains Research and Development Corporation (GRDC) and the Beijing Genomics Institute.

\section{Author details}

${ }^{1}$ Department of Agriculture, Fisheries \& Forestry (DAFF), Warwick, QLD, Australia. ${ }^{2}$ BGI-Shenzhen, Shenzhen, China. ${ }^{3}$ DAFFQ, Cooper's Plains, Brisbane, QLD, Australia. ${ }^{4}$ The University of Queensland, School of Agriculture and Food Sciences, Brisbane, QLD, Australia. ${ }^{5}$ The Institute of Molecular Biosciences, The University of Queensland, Brisbane, QLD, Australia. ${ }^{6}$ Queensland University of Technology, Brisbane, QLD, Australia. ${ }^{7}$ Department of Biology, University of Copenhagen, DK-2200 Copenhagen, Denmark. ${ }^{8}$ The Novo Nordisk Foundation Center for Basic Metabolic Research, University of Copenhagen, DK-2200 Copenhagen, Denmark. ${ }^{9}$ Queensland Alliance for Agriculture and Food Innovation, The University of Queensland, Warwick, QLD, Australia.

Received: 23 April 2014 Accepted: 20 September 2014 Published online: 26 September 2014

\section{References}

1. Borlaug NE: Feeding a world of 10 billion people: the miracle ahead. In Vitro Cell Dev Biol Plant 2002, 38:221-228.

2. Christou P, Twyman RM: The potential of genetically enhanced plants to address food insecurity. Nutr Res Rev 2004, 17:23-42.

3. Oerke EC: Crop losses to pests. J Agric Sci 2006, 144:31-43.

4. Yang S, Feng Z, Zhang X, Jiang K, Jin X, Hang Y, Chen J-Q, Tian D: Genome-wide investigation on the genetic variations of rice disease resistance genes. Plant Mol Biol 2006, 62:181-193. 
5. McHale L, Tan X, Koehl P, Michelmore RW: Plant NBS-LRR proteins: adaptable guards. Genome Biol 2006, 7:212.

6. Yue J-X, Meyers BC, Chen J-Q, Tian D, Yang S: Tracing the origin and evolutionary history of plant nucleotide-binding site-leucine rice repeat (NBS-LRR) genes. New Phytol 2012, 193:1049-1063. 2011

7. Zhou T, Wang Y, Chen J-Q, Araki H, Jing Z, Jiang K, Shen J, Tian D: Genome-wide identification of NBS genes in japonica rice reveals significant expansion of divergent non-TIR NBS-LRR genes. Mol Genet Genomics 2004, 271:402-415.

8. Monosi B, Wisser RJ, Pennill L, Hulbert SH: Full-genome analysis of resistance gene homologues in rice. Theor App/ Genet 2004, 109:1434-1447.

9. Hammond-Kosack KE, Jones JD: Plant disease resistance genes. Annu Rev Plant Physiol Plant Mol Biol 1997, 48:575-607.

10. Tan S, Wu S: Genome wide analysis of nucleotide-binding site disease resistance genes in Brachypodium distachyon. Comp Funct Genomics 2012, 418208: http://www.hindawi.com/journals/ijg/2012/418208/.

11. Meyers BC, Kozik A, Griego A, Kuang $H$, Michelmore RW: Genome-wide analysis of NBS-LRR-encoding genes in Arabidopsis. Plant Cell 2003, 15:809-834.

12. Yang S, Zhang X, Yue J-X, Tian D, Chen J-Q: Recent duplications dominate NBS-encoding gene expansion in two woody species. Mol Genet Genomics 2008, 280:187-198.

13. Paterson AH, Bowers JE, Bruggmann R, Dubchak I, Grimwood J, Gundlach $H_{\text {, }}$ Haberer G, Hellsten U, Mitros T, Poliakov A, Schmutz J, Spannagl M, Tang H, Wang X, Wicker T, Bharti AK, Chapman J, Feltus FA, Gowik U, Grigoriev IV, Lyons E, Maher CA, Martis M, Narechania A, Otillar RP, Penning BW, Salamov AA, Wang Y, Zhang L, Carpita NC, et al: The Sorghum bicolor genome and the diversification of grasses. Nature 2009, 457:551-556.

14. Tan X, Wang X, Wang Z, Li J, Paterson AH: Genomic-level comparison of NBS gene evolution in Zea mays and Sorghum bicolor [abstract]. Plant Anim Genome Conf XX 2012, [https://pag.confex.com/pag/xx/webprogram/Paper4080.html]

15. Li J, Ding J, Zhang W, Zhang Y, Tang P, Chen J-Q, Tian D, Yang S: Unique evolutionary pattern of numbers of gramineous NBS-LRR genes. Mol Genet Genomics 2010, 283:427-438

16. Bakker EG, Toomajian C, Kreitman M, Bergelson J: A genome-wide survey of R gene polymorphisms in Arabidopsis. Plant Cell 2006, 18:1803-1818.

17. Yang S, Li J, Zhang X, Zhang Q, Huang J, Chen J-Q, Hartl DL, Tian D: Rapidly evolving $\mathrm{R}$ genes in diverse grass species confer resistance to rice blast disease. Proc Natl Acad Sci U S A 2013, 110:18572-18577.

18. Yu J, Hu S, Wang J, Wong GK, Li S, Liu B, Deng Y, Dai L, Zhou Y, Zhang X, Cao M, Liu J, Sun J, Tang J, Chen Y, Huang X, Lin W, Ye C, Tong W, Cong L, Geng J, Han Y, Li L, Li W, Hu G, Huang X, Li W, Li J, Liu Z, Li L, et al: A draft sequence of the rice genome (Oryza sativa L. ssp. indica). Science 2002, 296:79-92.

19. Schnable PS, Ware D, Fulton RS, Stein JC, Wei F, Pasternak S, Liang C, Zhang J, Fulton L, Graves TA, Minx P, Reily AD, Courtney L, Kruchowski SS, Tomlinson C, Strong C, Delehaunty K, Fronick C, Courtney B, Rock SM, Belter E, Du F, Kim K, Abbott RM, Cotton M, Levy A, Marchetto P, Ochoa K, Jackson SM, Gillam B, et al: The B73 Maize genome: complexity, diversity, and dynamics. Science 2009, 326:1112-1115

20. Lai J, Li R, Xu X, Jin W, Xu M, Zhao H, Xiang Z, Song W, Ying K, Zhang M, Jiao $Y$, Ni P, Zhang J, Li D, Guo X, Ye K, Jian M, Wang B, Zheng H, Liang $H$, Zhang X, Wang S, Chen S, Li J, Fu Y, Springer NM, Yang Hm Wang J, Dai J, Schnable PS, Wang J: Genome-wide patterns of genetic variation among elite maize inbred lines. Nat Genet 2010, 42:1027-1030.

21. Xu X, Liu X, Ge S, Jensen JD, Hu F, Li X, Dong Y, Gutenkunst RN, Fang L, Huang L, Li J, He W, Zhang G, Zheng X, Zhang F, Li Y, Yu C, Kristiansen K, Zhang X, Wang J, Wright M, McCouch S, Nielsen R, Wang J, Wang W: Resequencing 50 accessions of cultivated and wild rice yields markers for identifying agronomically important genes. Nat Biotechno/ 2011, 30:105-111.

22. Hufford MB, Xu X, van Heerwaarden J, Pyhäjärvi T, Chia JM, Cartwright RA, Elshire RJ, Glaubitz JC, Guill KE, Kaeppler SM, Lai J, Morrell PL, Shannon LM, Song C, Springer NM, Swanson-Wagner RA, Tiffin P, Wang J, Zhang G, Doebley J, McMullen MD, Ware D, Buckler ES, Yang S, Ross-lbarra J: Comparative population genomics of maize domestication and improvement. Nat Genet 2012, 44:808-811.

23. Mace ES, Tai S, Gilding EK, Li Y, Prentis PJ, Bian L, Campbell BC, Hu W, Innes DJ, Han X, Cruickshank A, Dai C, Frère C, Zhang H, Hunt CH, Wang X, Shatte T, Wang M, Su Z, Li J, Lin X, Godwin ID, Jordan DR, Wang J: Whole genome sequencing reveals untapped genetic potential in Africa's indigenous cereal crop sorghum. Nat Commun 2013, 4:2320.

24. Scheideler M, Schlaich NL, Fellenberg K, Beissbarth T, Hauser NC, Vingron M, Slusarenko AJ, Hoheisel JD: Monitoring the switch from housekeeping to pathogen defense metabolism in Arabidopsis thaliana using CDNA arrays. J Biol Chem 2002, 277:10555-10561.

25. Wright SI, Charlesworth B: The HKA test revisited: a maximum-likelihood ratio test of the standard neutral model. Genetics 2004, 168:1071-1076.

26. Li P, Wang Y, Qian Q, Fu Z, Wang M, Zeng D, Li B, Wang X, Li J: LAZY1 controls rice shoot gravitropism through regulating polar auxin transport. Cell Res 2007, 17:402-410.

27. Furukawa T, Maekawa M, Oki T, Suda I, lida S, Shimada H, Takamure I, Kadowaki K: The Rc and Rd genes are involved in proanthocyanidin synthesis in rice pericarp. Plant J 2007, 49:91-102.

28. Martin T, Biruma M, Fridborg I, Okori P, Dixelius C: A highly conserved NB-LRR encoding gene cluster effective against Setosphaeria turcica in sorghum. BMC Plant Biol 2011, 11:151

29. Córdova-Campos O, Adame-Álvarez RM, Acosta-Gallegos JA, Heil M: Domestication affected the basal and induced disease resistance in common bean (Phaseolous vulgaris). Eur J Plant Pathol 2012, 134:367-379.

30. Bai J, Pennill LA, Ning J, Lee SW, Ramalingam J, Webb CA, Zhao B, Sun Q, Nelson JC, Leach JE, Hulbert SH: Diversity in nucleotide binding siteleucine rich repeat genes in cereals. Genome Res 2002, 12:1871-1884

31. Wisser RJ, Sun Q, Hulbert SH, Kresovich S, Nelson RJ: Identification and characterisation of regions of the rice genome associated with broadspectrum, quantitative disease resistance. Genetics 2005, 169:2277-2293.

32. Kang YJ, Kim KH, Shim S, Yoon MY, Sun S, Kim MY, Van K, Lee S-H: Genomewide mapping of NBS-LRR genes and their association with disease resistance in soybean. BMC Plant Biol 2012, 12:139-152.

33. Klein RR, Rodriguez-Herrera R, Schlueter JA, Klein PE, Yu ZH, Rooney WL: Identification of genomic regions that affect grain-mold incidence and other traits of agronomic importance in sorghum. Theor Appl Genet 2001, 102:307-319.

34. Mohan SM, Madhusudhana R, Mathur K, Howarth CJ, Srinivas G, Satish K, Reddy RN, Seetharama N: Co-localization of quantitative trait loci for foliar disease resistance in sorghum. Plant Breeding 2009, 128:532-535.

35. Mohan SM, Madhusudhana R, Mathur K, Chakravarthi DVN, Rathore S, Reddy RN, Satish K, Srinivas G, Mani NS, Seetharama N: Identification of quantitative trait loci associated with resistance to foliar diseases in sorghum [Sorghum bicolor (L.) Moench]. Euphytica 2010, 176:199-211.

36. Upadhyaya HD, Wang YH, Sharma R, Sharma S: Identification of genetic markers linked to anthracnose resistance in sorghum using association analysis. Theor Appl Genet 2013, 126:1649-1657.

37. McMullen MD, Simcox KD: Genomic organisation of disease and insect resistance genes in maize. Mol Plant Microbe Interact 1995, 8:811-815.

38. Gebhardt C, Valkonen JP: Organisation of genes controlling disease resistance in the potato genome. Annu Rev Phytopathol 2001, 39:79-102.

39. Cheng Y, Li X, Jiang H, Ma W, Miao W, Yamada T, Zhang M: Systematic analysis and comparison of nucleotide-binding site disease resistance genes in maize. FEBS J 2012, 279:2431-2443.

40. Zhang R, Murat F, Pont C, Langin T, Salse J: Paleo-evolutionary plasticity of plant disease resistance genes. BMC Genomics 2014, 15:187.

41. Cheng X, Jiang H, Zhao Y, Qian Y, Zhu S, Cheng B: A genomic analysis of disease-resistance genes encoding nucleotide binding sites in Sorghum bicolor. Genet Mol Biol 2010, 33:292-297.

42. Eckardht NA: A microRNA cascade in plant defense. Plant Cell 2012, 24:840.

43. Li F, Pignatta D, Bendix C, Brunkard JO, Cohn MM, Tung J, Sun H, Kumar P, Bakker B: MicroRNA regulation of plant innate immune receptors. Proc Natl Acad Sci U S A 2012, 109:1790-1795.

44. Luo S, Zhang Y, Hu Q, Chen J, Li K, Lu C, Liu H, Wang W, Kuang H: Dynamic nucleotide-binding-site and leucine-rich-repeat encoding genes in the grass family. Plant Physiol 2012, 159:197-210.

45. Goff SA, Ricke D, Lan TH, Presting G, Wang R, Dunn M, Glazebrook J, Sessions A, Oeller P, Varma H, Hadley D, Hutchison D, Martin C, Katagiri F, Lange BM, Moughamer T, Xia Y, Budworth P, Zhong J, Miguel T, Paszkowski U, Zhang S, Colbert M, Sun WL, Chen L, Cooper B, Park S, Wood TC, Mao L, Quail P, et al: A draft sequence of the rice genome (Oryza sativa L. ssp. japonica). Science 2002, 296:92-100.

46. Holub EB: The arms race is ancient history in Arabidopsis, the wildflower. Nat Rev Genet 2001, 2:516-527.

47. Larkin MA, Blackshields G, Brown NP, Chenna R, McGettigan PA McWilliam H, Valentin F, Wallace IM, Wilm A, Lopez R, Thompson JD, Gibson TJ, Higgins DG: Clustal W and Clustal $X$ version 20. Bioinformatics 2007, 23:2947-2948 
48. Tamura K, Peterson D, Peterson N, Stecher G, Nei M, Kumar S: MEGA5: Molecular evolutionary genetics analysis using maximum likelihood, evolutionary distance and maximum parsimony methods. Mol Biol Evol 2011, 28:2731-2739.

49. Perrier X, Jacquemoud-Collet JP: DARwin software. In 2006 [http://darwin.cirad.fr/]

50. Schnable JC, Freeling M, Lyons E: Genome-wide analysis of syntenic gene deletion in the grasses. Genome Biol Evol 2012, 4:265-277.

51. Kryzwinski MI, Schein JE, Birol I, Connors J, Gascoyne R, Horsman D, Jones SJ, Marra MA: Circos: an information aesthetic for comparative genomics. Genome Res 2009, 19:1639-1645.

52. Zhang Z, Li J, Zhao XQ, Wang J, Wong GK, Yu J: KaKs calculator: Calculating Ka and Ks through model selection and model averaging. Genomics Proteomics Bioinformatics 2006, 4:259-263.

53. Bandelt HJ, Forster $P$, Rãhl A: Median-joining networks for inferring intraspecific phylogenies. Mol Biol Evol 1999, 16:37-48.

54. Mace ES, Jordan DR: Integrating sorghum whole genome sequence information with a compendium of sorghum QTL studies reveals uneven distributions of QTL and of gene-rich regions with significant implications for crop improvement. Theor Appl Genet 2011, 123:169-191.

55. Mace ES, Jordan DR: Location of major effect genes in sorghum (Sorghum bicolor (L.) Moench). Theor Appl Genet 2010, 121:1339-1356.

56. Upadhyaya HD, Wang YH, Sharma R, Sharma S: SNP markers linked to leaf rust and grain mold resistance in sorghum. Mol Breeding 2013, 32:451-462.

doi:10.1186/s12870-014-0253-z

Cite this article as: Mace et al:: The plasticity of NBS resistance genes in sorghum is driven by multiple evolutionary processes. BMC Plant Biology 2014 14:253

\section{Submit your next manuscript to BioMed Central and take full advantage of:}

- Convenient online submission

- Thorough peer review

- No space constraints or color figure charges

- Immediate publication on acceptance

- Inclusion in PubMed, CAS, Scopus and Google Scholar

- Research which is freely available for redistribution 